\title{
Neural Network Study of the Effect of Corona Discharge Treatment on the Surfaces of Mechanical Engineering Products
}

\author{
Sergiy V. Kovalevskyy, Ivan N. Starodubcev \\ Donbas State Engineering Academy (DSEA), Faculty Integrated Tenology And Equipment, Kramatorsk, Ukraine \\ Email address: \\ kovalevskii@i.ua (S. V. Kovalevskyy), s_t_a_r_13@mail.ru (I. N. Starodubcev)
}

\section{To cite this article:}

Sergiy V. Kovalevskyy, Ivan N. Starodubcev. Neural Network Study of the Effect of Corona Discharge Treatment on the Surfaces of Mechanical Engineering Products. American Journal of Neural Networks and Applications. Vol. 1, No. 2, 2015, pp. 48-51.

doi: 10.11648/j.ajnna.20150102.14

\begin{abstract}
We suggest a method of surface saturation in the corona discharge, which is saturated with metal ions (tungsten, aluminum, copper, etc.), details of the surface layer due to the formation of carrier ions, namely the pinch corona discharge, which is being pulsed nature allows the process to flow in the air with self and with the help of surfactants.
\end{abstract}

Keywords: Ion Implantation, Corona Discharge, Neural Network, Surface Saturation, Surfactants, Micro-hardness

\section{Introduction}

Modification of the surface layer of detail applied to improve the performance of surfaces of machine parts, molds, equipment and tools are widely used in modern engineering. The treatment used to improve the wear resistance of parts operating under conditions of adhesion and diffusion wear at high ambient temperatures perform mainly in vacuum systems.

The widespread industrial use of hardfacing can solve a range of essential tasks:

- a significant increase in the strength and reliability of parts;

- Increased service life of the working surfaces of parts;

- reducing the consumption of expensive materials and scarce items.

The most promising method to obtain hard and wearresistant coatings is modified by ion implantation (Fig. 1), which provides coverage in the nano-crystalline states, which is characterized by high physical processing properties.

Ion implantation - is the introduction to the solid surface of almost any ion, pre-overclocked in the electromagnetic field. These ions are introduced into the material to a depth of 0.01 to a few tens of micrometers, creating a surface layer of a special structural state. The thickness of modified layer depends on the ion energy, mass and the mass of the target atoms. The ion energy can be varied within wide limits (depending on the properties of a combination of ion - target of several $\mathrm{KeV}$ to several $\mathrm{MeV}$. In addition the introduction of the ion in the target surface takes place without complying with the laws of classical thermodynamics, which determines the equilibrium processes such as diffusion. [1,2,3,9].

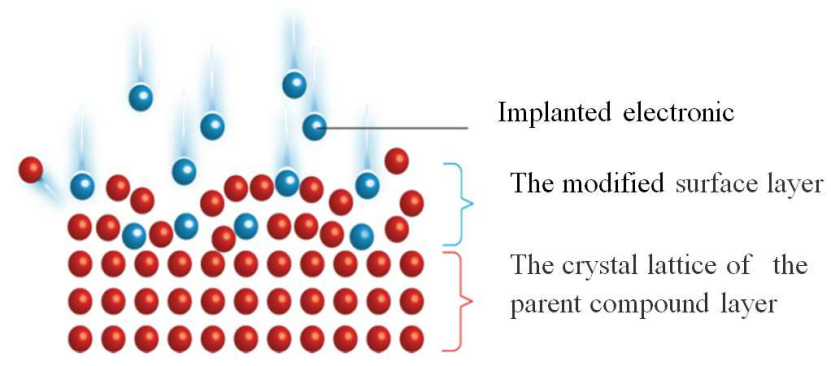

Figure 1. The process of ion implantation.

Plasma ion-implantation - the process of coating the surface of the part (product) through the plasma column. Pinch - is partially or fully ionized gas with the property and has electric heat $[4,10,11]$.

The essence of the method is to spray refractory metal cathode, the formation of ions in the low-temperature plasma and the bombardment of the surface to be strengthening. Cathode material evaporates in the form of high-speed jets (evaporation rate of $1 \times 106 \mathrm{~cm} / \mathrm{s}$ ), containing both charged and neutral particles. The jets are the plasma fluxes of atoms and ions with a high degree of ionization.

Coverage obtained by thermal evaporation, cathodic or ion - plasma spraying or ion bombardment of the surface 
material is deposited. As a reactive gas is used, nitrogen or carbon. According to the coating consists of a nitride or carbide compounds of refractory metals. The method of thermal evaporation can be carried out without the oxidative atmosphere of neutral gas or in air. The essential difference lies in the different methods produced a plasma in a plasma having a neutral or charged ionized component $[5,6,7,8]$.

\section{Basic Part}

The proposed model of treatment by ion-plasma implantation:

- In the environment of the corona discharge;

- In the environment of the corona discharge using a surface-active substances;

- By regulating the automaticity of the gap.

Allow to obtain the following results on the effects of the surface layers of machine parts, in different environments and different materials, electrodes.

The essence of this method consist in the fact that we have proposed setting allows you to eliminate the gas chambers, the role of oxygen used by cable insulator corona discharge, which passed a current of high voltage.

Implementation of the saturation method by electron-ion implantation in an environment of corona discharge is carried out as follows.

The part which is to be processed (body rotation), mounted on a lathe, but for the experiments in detail sufficient to establish the centers of the experimental setup. From the source of high voltage electric current flow creates a corona discharge, which affects the surface of the workpiece. In the body of the cord brings high-voltage electrode is in agreement polarity high-voltage cable. The electrode, which is installed in the holder with the PCB and inside there is a corona discharge, detail, in turn, makes a rotational movement, as a result, processing, and held a working surface of the part.

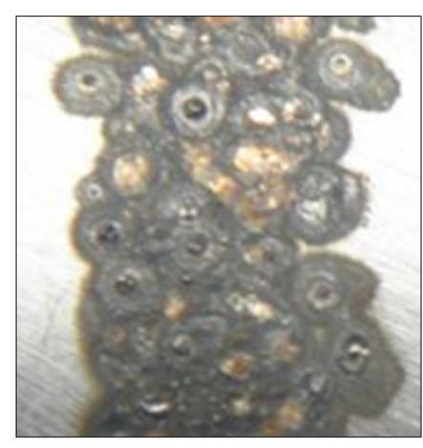

a)

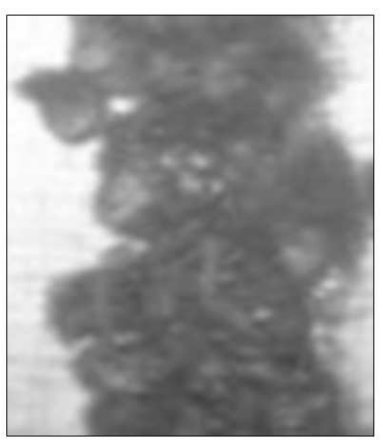

b)
Figure 2. A sample treated with the saturation of the type: a - when you turn on the installation with direct polarity, $b-$ when you turn on the installation of reverse polarity.

Treatment was carried out with forward and reverse polarity to investigate the effect of implantation. And it was noted that the straight polarity (electrode "plus" part "minus"), there was the transfer of ions from the electrode on the surface of the sample (Fig. 2a), and reverse polarity (electrode - "minus" part - " plus "), is the erosion of the sample surface (Fig. 2b)

The same process was carried out at different conditions by varying the voltage and changing the duration of treatment. As a result, treatment was carried out as details of the appearance of an arc (Fig. 3 b), obtained as a result of the breakdown voltage rises, and in the absence of the arc (Fig. 3a), where the transfer of ions was carried out by a potential difference.

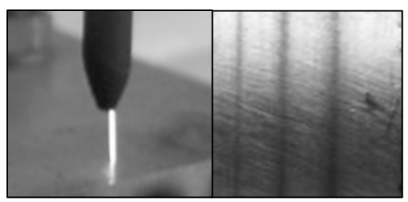

a)

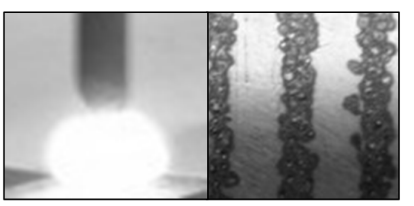

b)
Figure 3. Sample treated with saturation: $a$ - without an arc discharge, $b$ in an arc discharge.

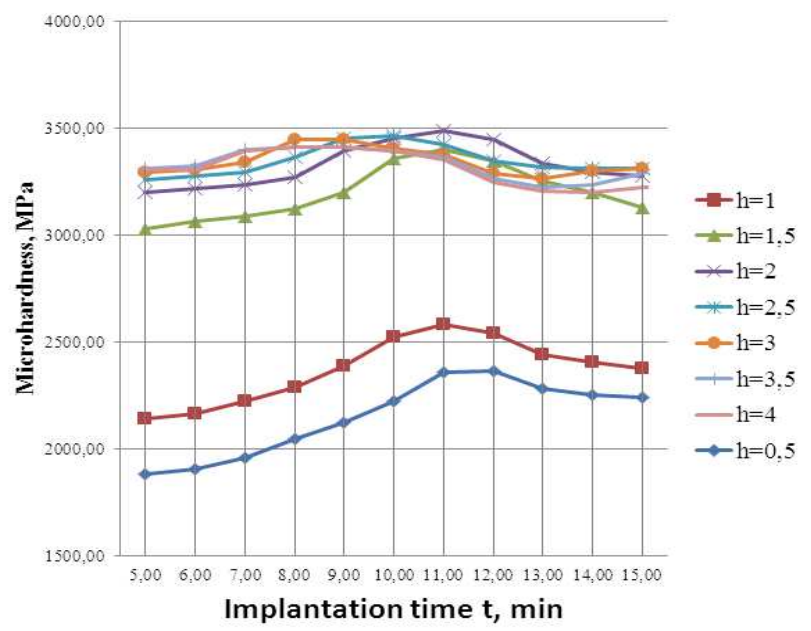

Figure 4. Dependence of micro hardness of the processing time for different values of the gap (if there is an arc).

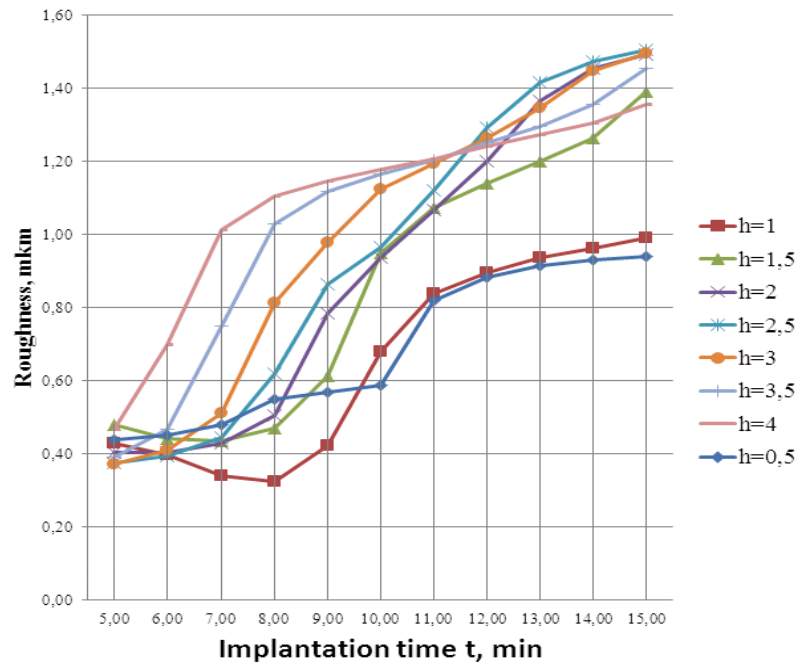

Figure 5. A plot of the roughness of the processing time for different values of the gap (if there is an arc). 


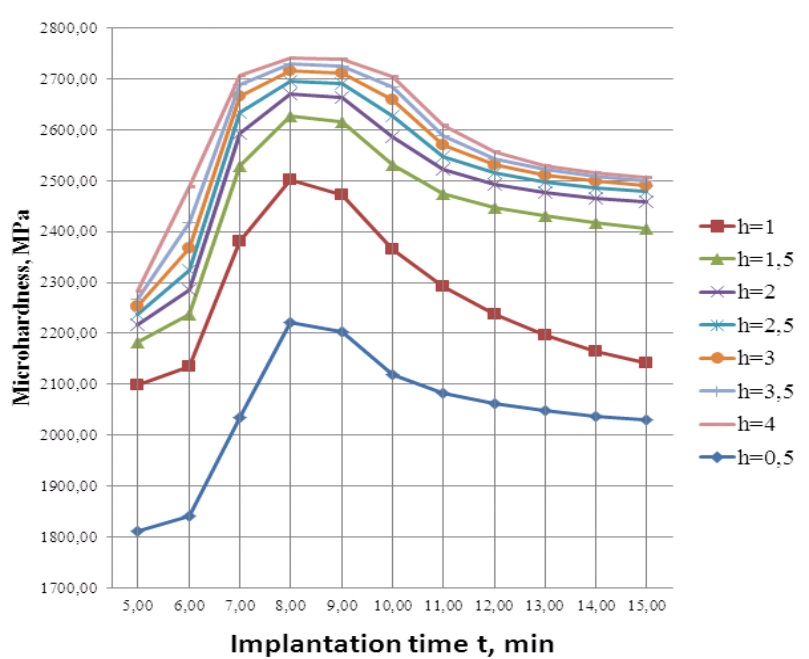

Figure 6. Dependence of microhardness on the processing time for different values of the gap (without straps).

On the basis of data obtained using neural network modeling have been analyzed a new way of working surfaces of the saturation of machine electron-ion implantation, based on a verbal description (neural network) of the two mathematical models of integrated data processing performance of the microhardness and roughness, can investigate the effect of treatment regimes on the quality and performance of the machined surface are on (Fig. 4, 5, 6).

Obviously, when using the method of electron-ion implantation are expanding technological possibilities of its use, increased convenience and ease of use under normal operating conditions. Experimentally confirmed that the processing modes, namely, the voltage and duration, affecting the quality and characteristics of the treated surface. The results indicate that the improved operational performance of working surfaces of parts.

So, we came to the conclusion that the need for more indepth and thorough investigation to continue this method to more clearly show the obvious advantages and the need to use it in production.

For the case with the potential of accelerated with the use of surfactants perform analysis of the relationship of the material implanted electrodes and the time of implantation with the indicators of quality and micro-hardness of the surface layer of the machined surface in the form of graphs, based on data from neural network modeling. Before processing the material he had previously covered by a surfactant that allows you to improve the properties of the surface layer of workpieces machines. This allows us to determine the optimal time and material, under which achieved the desired micro-hardness and surface quality are shown in (Fig. 7, 8).

Of the constructed image of dependencies (Fig. 7 and Fig. 8 ) we see that the smallest roughness of $\mathrm{Ra}=0,218953$ micrometers is achieved using an accelerated building, using $\mathrm{Al}$ electrode implantation time $\mathrm{t}=5 \mathrm{~min}$.

Micro hardness of $\mathrm{HB}=3023.092 \mathrm{MPa}$ is achieved using high-voltage discharge, using the $\mathrm{W}$ electrode implantation time $\mathrm{t}=1 \mathrm{~min}$ (Fig. 7 and 8$)$.

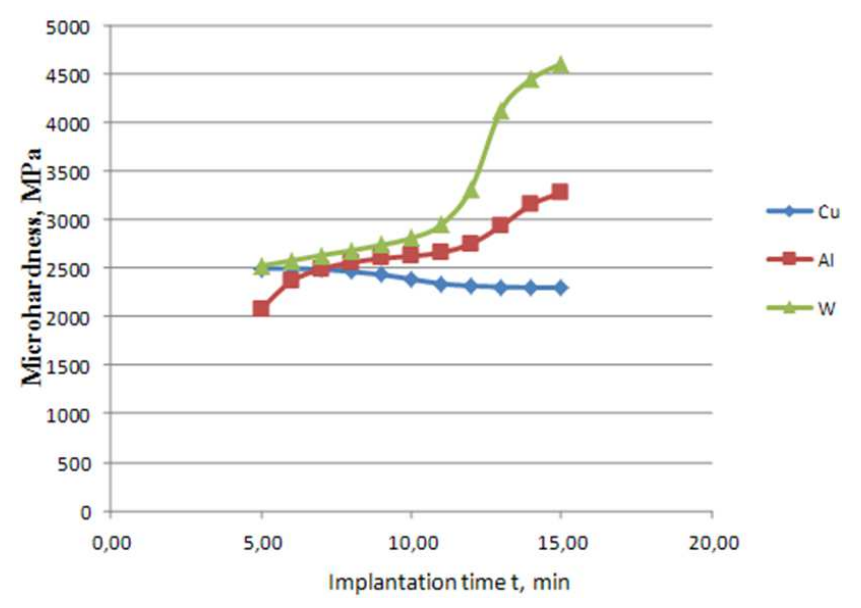

Figure 7. Dependence of micro hardness on the time of implantation.

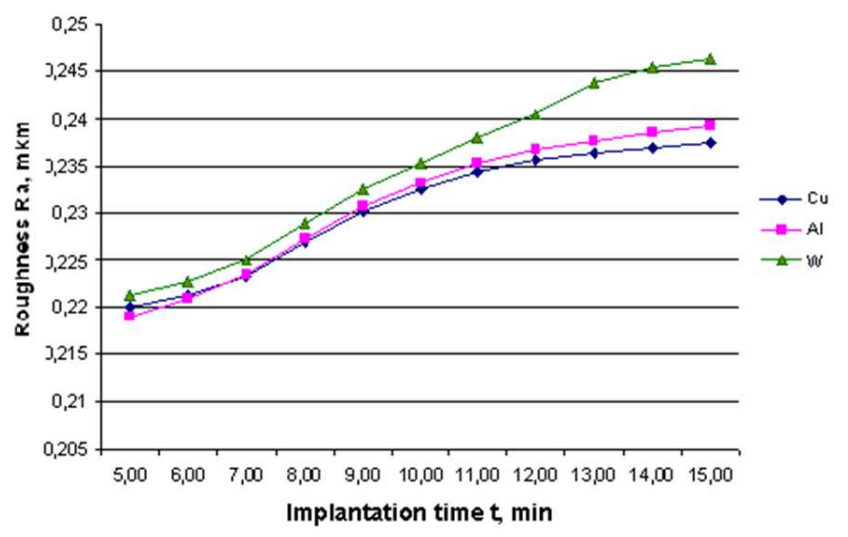

Figure 8. The dependence of roughness on the time of implantation.

In general, the graphical analysis of dependence, we can conclude that the use of pulsed electron-implantation leads to an increase in the microhardness of the surface layer of machine parts.

When processing an arc discharge with an excess of oxygen diffusion, due to high temperatures. To avoid overheating in the area of treatment there are several possible solutions.

The first is the use of a pulsed discharge. As a conductor, as previously, the cord will be used corona discharge, but it will create a passage of pulse current, which carries the flow of ions forming the electrode. As a result, the amendments eliminated overheating of the treatment area, due to impulsive action on the part surface, and combustion of carbon with the working surface of the part to be saturated, and from a material that is implanted. Second, the possibility of a saturation of the process of self-regulation, due to variable-length column of corona discharge.

Our experimental studies are based on the assumption of increasing the performance of the working surfaces of parts by means of surface saturation in the corona discharge with self-regulation process. Research also is subject to the quality of the surface, the resulting treatment.

Treatment was carried out with forward and reverse 
polarity to investigate the effect of saturation. And it was noted that the straight polarity (electrode "plus" part "minus") there was the transfer of ions from the electrode on the surface of the sample, and reverse polarity (electrode "minus" part - the "plus"), the erosion surface the sample.

\section{Conclusion}

Obviously, when using the method of electron-ion implantation are expanding technological possibilities of its use, increased convenience and ease of use under normal operating conditions. Experimentally confirmed that the processing modes, namely, the voltage and duration, affecting the quality and characteristics of the treated surface. The results indicate that the improved operational performance of working surfaces of parts.

When using the method of surface saturation in the corona discharge process with self expanding technological possibilities of its use, increased convenience and ease of use in normal operating conditions. Experimentally confirmed that the processing factors, namely, current, and material processing, affect the quality and characteristics of the treated surface. The results indicate that the improved operational performance of working surfaces of parts.

\section{References}

[1] Kadyrzhanov KK. Komarov FF. Pogrebnyak AD, VS Rusakov, TE Turkebaev Ion-beam-plasma nonno and modification of materials. Monograph. - Moscow: Moscow State University Press, 2005. - 640 p. ISBN 5-211-05153-X.

[2] Stepanova, T. Technology of surface hardening of machine parts: a training manual / T. Stepanov, Ivan. State. him.Tekhnol. University t-Ivanovo, 2009. - 64C.

[3] Andreev AA, Sable LP, Shulaev V., Grigoriev, SN Vacuum arc devices and coatings. Monograph. - Kharkov: NSC KIPT, 2005. - $236 \mathrm{p}$.
[4] Andreev AA, Sable LP, VM Shulaev Vacuum-arc plasma sources (evaporators) / / Mater. XI Int. Scientific and Technical. conference. "High technology industry in Russia" (Moscow). - 2005. - S. 566 - 587.

[5] Five. Kostyuk, GI, A. Voloshko. Guly SV, etc. The influence of a stationary high-frequency discharge on the formation of coatings applied by the IRB / / Vacuum processes for fabricating thin films and coatings: Kharkov, 2001. - page 239, 250.

[6] Maltsev, P., et al, "Nanotechnology. Nanomaterials. Nanosystem engineering ", Moscow, Vol. Technosphere, 2008, 430 p. ISBN 978-5-94836-180-2

[7] Comparative analysis of modern systems of automated designing of machines on kokhonena's cards / V. Fedorinov, S. Kovalevskyy // International conference "Research and Development in Mechanical Industrry" RaDMI 2010, 16-19 Septmber. - Vrnjcka Banja,Serbia, 2010. - Vol.1. - P. 19-32.

[8] Research of technological possibilities of management of technological system dynamic descriptions / S. Kovalevskyy, O. Kovalevska, T. Fedyuk, I. Starodubcev // 11th International conference "Research and Development in Mechanical Industry" RaDMI 2011, 15-18 September. - Sokobanja, Serbia, 2011. - Vol. 1. - P. 224-229.

[9] Perfection of non-destructive methods of control of surfaces of details of machines / S. Kovalevskyy, O. Kovalevska, A. Kostina // 11th International conference "Research and Development in Mechanical Industry" RaDMI 2011, 15-18 September. - Sokobanja, Serbia, 2011. - Vol. 1. - P. 230-235.

[10] Investigation with the application of neural networks ability surface processing working parts machine on wednesday corona discharge / S. Kovalevskyy, I. Starodubcev, S. Sokur // 12th International conference "Research and Development in Mechanical Industrry" RaDMI 2012, 13-17 September. Vrnjcka Banja, Serbia, 2012. - Vol. 1. - P. 235-241.

[11] Neural network analysis of natural frequencies spectrum of products for the diagnosis of their parameters / S. Kovalevskyy, I. Starodubcev // 12th International conference "Research and Development in Mechanical Industrry" RaDMI 2012, 13-17 September. - Vrnjcka Banja, Serbia, 2012. - Vol. 1. - P. 230-234. 